\title{
ALMA and NACO observations towards the young exoring transit system J1407 (V1400 Cen)^
}

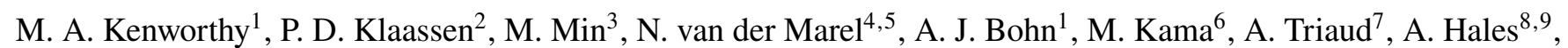
J. Monkiewicz ${ }^{10}$, E. Scott ${ }^{11}$, and E. E. Mamajek ${ }^{12,13}$

${ }^{1}$ Leiden Observatory, Leiden University, PO Box 9513, 2300 RA Leiden, The Netherlands

e-mail: kenworthy@strw.leidenuniv.nl

${ }^{2}$ UK Astronomy Technology Centre, Royal Observatory Edinburgh, Blackford Hill, Edinburgh EH9 3HJ, UK

${ }^{3}$ SRON Netherlands Institute for Space Research, Sorbonnelaan 2, 3584 CA Utrecht, The Netherlands

${ }^{4}$ Herzberg Astronomy \& Astrophysics Programs, National Research Council of Canada Herzberg, 5071 West Saanich Rd, Victoria, BC, V9E 2E7, Canada

${ }^{5}$ Department of Physics \& Astronomy, University of Victoria, Victoria, BC, V8P 1A1, Canada

${ }^{6}$ Institute of Astronomy, University of Cambridge, Madingley Road, Cambridge, CB3 OHA, UK

${ }^{7}$ School of Physics \& Astronomy, University of Birmingham, Edgbaston, Birmingham B15 2TT, UK

${ }^{8}$ National Radio Astronomy Observatory, 520 Edgemont Road, Charlottesville, VA 22903-2475, USA

9 Atacama Large Millimeter/Submillimeter Array, Joint ALMA Observatory, Alonso de Cordova 3107, Vitacura 763-0355, Santiago, Chile

${ }^{10}$ School of Earth and Space Exploration, Arizona State University, Tempe, AZ 85287, USA

${ }^{11}$ Chinese Academy of Sciences, 52 Sanlihe Road, Xicheng District, 100864 Beijing, PR China

12 Jet Propulsion Laboratory, California Institute of Technology, M/S 321-100, 4800 Oak Grove Drive, Pasadena, CA 91109, USA

${ }^{13}$ Department of Physics \& Astronomy, University of Rochester, Rochester, NY 14627, USA

Received 20 June 2019 / Accepted 16 November 2019

\begin{abstract}
Aims. Our aim was to directly detect the thermal emission of the putative exoring system responsible for the complex deep transits observed in the light curve for the young Sco-Cen star 1SWASP J140747.93-394542.6 (V1400 Cen, hereafter J1407), confirming it as the occulter seen in May 2007, and to determine its orbital parameters with respect to the star.

Methods. We used the Atacama Large Millimeter/submillimeter Array (ALMA) to observe the field centred on J1407 in the $340 \mathrm{GHz}$ (Band 7) continuum in order to determine the flux and astrometric location of the ring system relative to the star. We used the VLT/NACO camera to observe the J1407 system in March 2019 and to search for the central planetary mass object at thermal infrared wavelengths.

Results. We detect no point source at the expected location of J1407, and derive an upper limit $3 \sigma$ level of $57.6 \mu \mathrm{Jy}$. There is a point source detected at an angular separation consistent with the expected location for a free-floating ring system that occulted J1407 in May 2007, with a flux of $89 \mu \mathrm{Jy}$ consistent with optically thin dust surrounding a massive substellar companion. At $3.8 \mu \mathrm{m}$ with the NACO camera, we detect the star J1407 but no other additional point sources within 1.3 arcsec of the star, with a lower bound on the sensitivity of $6 M_{\mathrm{Jup}}$ at the location of the ALMA source, and down to $4 M_{\mathrm{Jup}}$ in the sky background limit.

Conclusions. The ALMA upper limit at the location of J1407 implies that a hypothesised bound ring system is composed of dust smaller than $1 \mathrm{~mm}$ in size, implying a young ring structure. The detected ALMA source has multiple interpretations, including: (i) it is an unbound substellar object surrounded by warm dust in Sco-Cen with an upper mass limit of $6 M_{\mathrm{Jup}}$, or (ii) it is a background galaxy.
\end{abstract}

Key words. stars: pre-main sequence - submillimeter: planetary systems - planets and satellites: formation - protoplanetary disks planet-disk interactions - planets and satellites: gaseous planets

\section{Introduction}

Planet formation theory predicts that gas giant planets form in circumstellar disks (CSDs) which disperse on a timescale of 2-3 Myr (Williams \& Cieza 2011). Once a planetary embryo forms within the CSD, a circumplanetary disk (CPD) builds inside the gravitational domain of the embryo, and gas and dust are transported through this disk and accrete onto the planet (see reviews by e.g. Armitage 2011; Kley \& Nelson 2012). Once the CSD disperses, the remaining gaseous CPD accretes onto the

\footnotetext{
${ }^{\star}$ Reduced images are only available at the CDS via anonymous ftp to cdsarc.u-strasbg. fr (130.79.128.5) or via http://cdsarc. u-strasbg.fr/viz-bin/cat/J/A+A/633/A115
}

planet in a short time (Szulágyi et al. 2014; Perez et al. 2015) or possibly on longer timescales depending on the planet formation mechanism (Szulágyi et al. 2017) and may form moons within the Hill radius of the planet (Canup \& Ward 2002). The duration of this intermediate stage is not strongly constrained by observations (upper limits of millimetre-sized particles to CPDs around known exoplanets are reported in Pérez et al. 2019), but the large solid angle subtended by the Hill sphere filling disk and rings imply that transits of these systems may be common around young stars.

1SWASP J140747.93-394542.6 (hereafter J1407) is a premain-sequence, $\sim 16 \mathrm{Myr}$ old, $0.9 M_{\odot}, V=12.3 \mathrm{mag}$ K5 star with parallax 7.1835 \pm 0.0447 mas (Gaia Collaboration 2018), 
consistent with $d=138.7 \pm 0.6 \mathrm{pc}$ (Bailer-Jones et al. 2018) and membership of the Sco-Cen OB association (Mamajek et al. 2012). Mamajek et al. (2012) reported light curves for J1407 from the Super Wide Angle Search for Planets (SuperWASP; Butters et al. 2010) and the All Sky Automated Survey (ASAS; Pojmanski 2002). These light curves show that the star underwent a complex series of deep transits lasting 56 days around 2007 May and includes a maximum dimming of $>95 \%$. The gradient of the light curve during the eclipse in 2007 can be used to derive a transverse velocity of about $35 \mathrm{~km} \mathrm{~s}^{-1}$; combined with the 56 day duration, this leads to a diameter of the order of $1.2 \mathrm{au}$, marginally consistent with a Hill sphere filling structure around a substellar companion to J1407. An inversion of the light curve suggests several dozen rings with at least one cleared gap suggesting the presence of a forming exomoon (Mamajek et al. 2012; van Werkhoven et al. 2014; Kenworthy \& Mamajek 2015). Subsequent papers have detailed the search for successive eclipses in photometric data (Kenworthy \& Mamajek 2015; Mentel et al. 2018) through radial velocity and direct imaging with full aperture and non-redundant mask imaging (Kenworthy et al. 2015) that have placed successively more stringent limits on the mass and orbital period of the putative companion. Circular orbits are strongly ruled out with these studies and the diameter of the ring system model, along with the detailed smallscale structure deduced from nightly flux variations, makes it very challenging to explain the apparent stability and coherence of the rings (Zanazzi \& Lai 2017; Rieder \& Kenworthy 2016). Another challenge is whether the ring gaps are caused by mean-motion resonances (MMRs) or by direct clearing, and the elliptical orbit at periastron disfavours the MMR hypothesis (Sutton 2019). With the central massive object undetected, we turn to detecting the thermal radiation from the rings themselves. The rings are estimated to be $1.2 \mathrm{au}$ in diameter based on the model described in Kenworthy \& Mamajek (2015).

In this paper we present data from the Atacama Large Millimeter/submillimeter Array (ALMA) taken in 2017 in a search for thermal emission from the rings of the J1407 occulting object. We report a detection of a source consistent with millimetre-sized material close to the location of the star with ALMA, but we detect no point source at thermal infrared wavelengths at the location of the ALMA source. The non-detection in the thermal infrared is consistent with an upper mass limit of $6 M_{\text {Jup }}$ at the age of the Sco-Cen association. The separation of the detected source from the location of J1407 is consistent with the location of a free-floating object with rings that has moved at a constant velocity (derived from the original eclipse with a value of $35 \mathrm{~km} \mathrm{~s}^{-1}$ ) since the eclipse seen in 2007 .

In Sects. 2 and 3 we describe the observations with ALMA and NACO and the subsequent data reduction. The interpretation is dependent on the astrometry, and this is discussed in detail in Sect. 4. In the last sections we discuss the hypotheses for this detection and future observations that will constrain the nature of this source.

\section{Observations with ALMA}

The star J1407 was observed by ALMA on UT 20, 22, and 24 July 2017 for a total of $2 \mathrm{~h}$ on source and a total time of $5.11 \mathrm{~h}$. Bandpass, phase, and flux calibrations were all carried out using J1427-4206, which had a flux between 1.46 and $1.5 \mathrm{Jy}$ in the observed spectral windows (at 336.495, 338.432, 348.495 , and $350.495 \mathrm{GHz}$ ). The observations were made in time domain mode (TDM) with 128 channels per spectral window. The mean atmospheric precipitable water vapour (pwv) content was between 0.5 and $0.86 \mathrm{~mm}$ across the observations, as summarised in Table 1. Similarly, the minimum and maximum baseline lengths for each observation is presented in the same table.

All ALMA data in this paper were reduced using CASA 4.7.2 (McMullin et al. 2007). The data were originally pipeline reduced, with a secondary phase calibrator (J1405-3907) used in a pre-calibration gain table to aid the gain calibration of the primary phase calibrator. However, this secondary calibrator was later found to be a double source. This could increase the uncertainty of the phase calibration, and thus the astrometric uncertainties. To control for this uncertainty, the double source secondary calibrator was flagged out of the dataset and explicitly removed from the calibration process (in the first hifa_flagdata() pipeline step), and the pipeline was re-run without it. The calibration and final products were unchanged, but any astrometric uncertainty introduced by having an extended phase calibrator was removed. Self-calibration of the final dataset was not possible as the target was too faint. The final calibrated data was imaged using natural weighting, which resulted in a synthesised beam of $0.12 \times 0.07^{\prime \prime}$ at a position angle of $-77.5^{\circ}$.

A point source was detected in the ALMA field near the expected location of J1407 (see Fig. 1). As the point source was near the phase centre of the observations, we did not do any primary beam correction. Using the CASA task imfit, we find that the source has a position of $\alpha=14: 07: 47.9311( \pm 0.00062)$, $\delta=-39: 45: 43.26068\left( \pm 0^{\prime \prime} .00388\right)($ ICRS $)$, and a size of $(95.1 \pm$ $18.1) \times(58.4 \pm 6.1)$ mas at a position angle of $113^{\circ} .4 \pm 8^{\circ} .4$, which means it is unresolved in our synthesised beam. Using the CASA task imstat, we derive a peak flux of $89 \mu \mathrm{Jy} \mathrm{beam}^{-1}$ and an rms noise of $19 \mu \mathrm{Jy}_{\text {beam }}{ }^{-1}$, resulting in a $4.7 \sigma$ detection. We take the J1407 stellar parameters to be $T_{*}=4500 \mathrm{~K}$, $R_{*}=1.039 \pm 0.075 R_{\odot}$, and $L_{*}=0.40 \pm 0.02 L_{\odot}$ at a distance $d=139 \mathrm{pc}$ and a frequency of $345 \mathrm{GHz}$. The stellar photosphere flux $F_{*}$ from $\mathrm{J} 1407$ is therefore

$F_{*}=B_{v}\left(T_{*}\right) \pi R_{*}^{2} / d^{2}=1.5 \mu \mathrm{Jy}$.

This is much fainter than the detection limit of our observation, and so the star is not expected to be visible in our image.

\section{Observations with NACO}

The star J1407 was observed with VLT/NACO (Lenzen et al. 2003; Rousset et al. 2003) on 1 March 2019 UT at a wavelength of $3.8 \mu \mathrm{m}$. The observing conditions were excellent with an average seeing of $0.46 \pm 0.08$ and a coherence time of $5.66 \pm 1.12$ millisec. The data were obtained in cube burst mode, with telescope pupil tracking (Kasper et al. 2009) enabled to minimise the effect of residual speckles from the telescope optics for optimal Angular Differential Imaging (ADI; Marois et al. 2006).

Excluding telescope and instrument overheads the on-source integration was $5220 \mathrm{~s}$ with a field rotation of 71.8 . The star J1407 was unsaturated in the images and acted as a PSF reference for subsequent data reduction and fake companion injection. Processing the data with the latest release of the PynPoint package (Stolker et al. 2019) and applying Principal Component Analysis (PCA; Amara \& Quanz 2012) results in a sensitivity map for point sources. Close to the star, the sensitivity is dominated by noise from the stellar speckle halo, falling to the sky background level noise limit at larger angular separations. The processing is dependent on the number of PCA components used in the data reduction pipeline. A larger number of PCA components 
Table 1. Calibrators and observing parameters for ALMA project 2016.1.00470.S.

\begin{tabular}{|c|c|c|c|c|c|c|c|c|}
\hline \multirow[b]{2}{*}{ Execution } & \multirow{2}{*}{$\begin{array}{l}\text { Time on } \\
\text { science source } \\
(\mathrm{min})\end{array}$} & \multicolumn{3}{|c|}{ Calibrators } & \multirow[b]{2}{*}{ Observing date } & \multicolumn{2}{|c|}{ Baseline lengths } & \multirow{2}{*}{$\begin{array}{l}\text { Mean } \\
\text { PWV }\end{array}$} \\
\hline & & Phase & Bandpass & Flux & & $\begin{array}{l}\text { Max } \\
(\mathrm{m})\end{array}$ & $\begin{array}{l}\text { Min } \\
(\mathrm{m})\end{array}$ & \\
\hline X54fd & 40 & J1427-4206 & J1427-4206 & J1427-4206 & $2017-07-24$ & 3638 & 30.5 & 0.51 \\
\hline $\mathrm{X} 238 \mathrm{e}$ & 40 & J1427-4206 & J1427-4206 & J1427-4206 & $2017-07-20$ & 3697 & 16.7 & 0.86 \\
\hline Xd6d & 40 & J1427-4206 & J1427-4206 & J1427-4206 & 2017-07-22 & 3697 & 16.7 & 0.50 \\
\hline
\end{tabular}

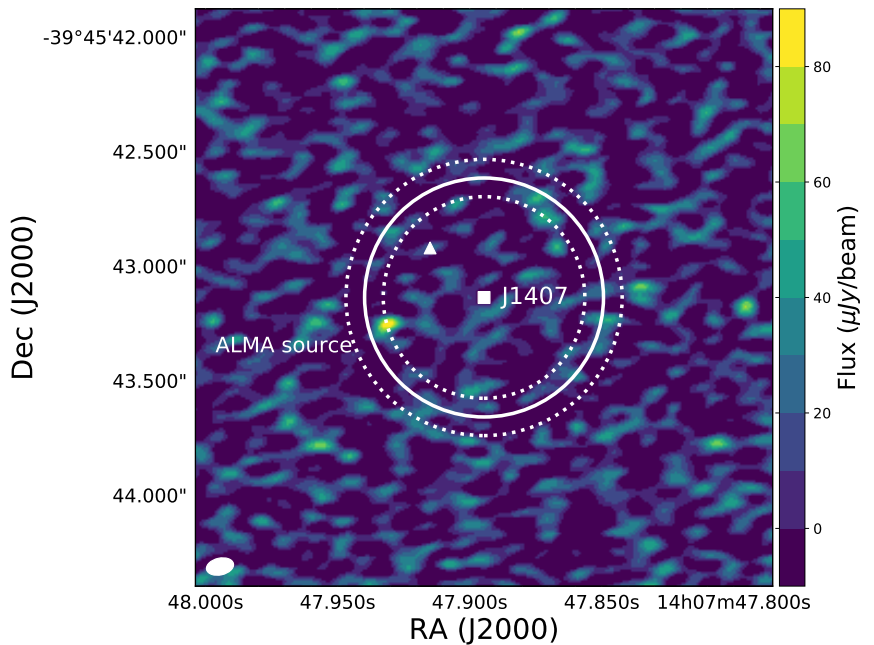

Fig. 1. ALMA image of the field towards J1407. The location of the star J1407 in 2017 is shown as a white square, and the white triangle gives the location at the time of the eclipse in 2007. The white ring indicates the expected location for an object travelling at $35 \mathrm{~km} \mathrm{~s}^{-1}$ between the two epochs (assuming that the object is co-distant with J1407); the dotted lines represent the $1 \sigma$ uncertainties. The detected ALMA source is at a position angle of approximately 95 degrees. The ALMA beam is shown in the lower left corner.

removes more speckles at small angular separations, but also removes flux from any point sources in the image. To correct for this systematic loss of companion flux, fake point sources are injected at several radii from the star into the data, and then the recovered flux is measured after the pipeline data reduction. The $5 \sigma$ contrast was calculated by injecting fake companions (created from the stellar PSF) at various angular separations (0.14-1.20 arcsec in 0.054 arcsec steps) and position angles (at 60 degree increments starting from 0 degrees). The small number of statistically independent patches close to the central star means that a correction is required to the sensitivity as described in Mawet et al. (2014), and we apply this correction to the contrast curve.

We determined that 5 PCA components is a reasonable compromise between speckle removal and sky background sensitivity, and the final image is shown in Fig. 2, which shows that the sensitivity is dominated by the noise from the sky background in all but the central 0.3 arcsec.

To convert from sensitivity in magnitudes to companion mass, we use the AMES-Cond (Baraffe et al. 2003; Allard et al. 2001) models for conversion of contrast to Jupiter masses and assume an age of $16 \mathrm{Myr}$ corresponding to the age of Sco-Cen, with the resultant contrast curve shown in Fig. 3. No point sources are detected within the field of view, down to $6 M_{\mathrm{Jup}}$ at $30 \mathrm{au}(0.25 \mathrm{arcsec})$ and dropping to less than $4 M_{\text {Jup }}$ beyond $100 \mathrm{au}(0.70 \mathrm{arcsec})$.

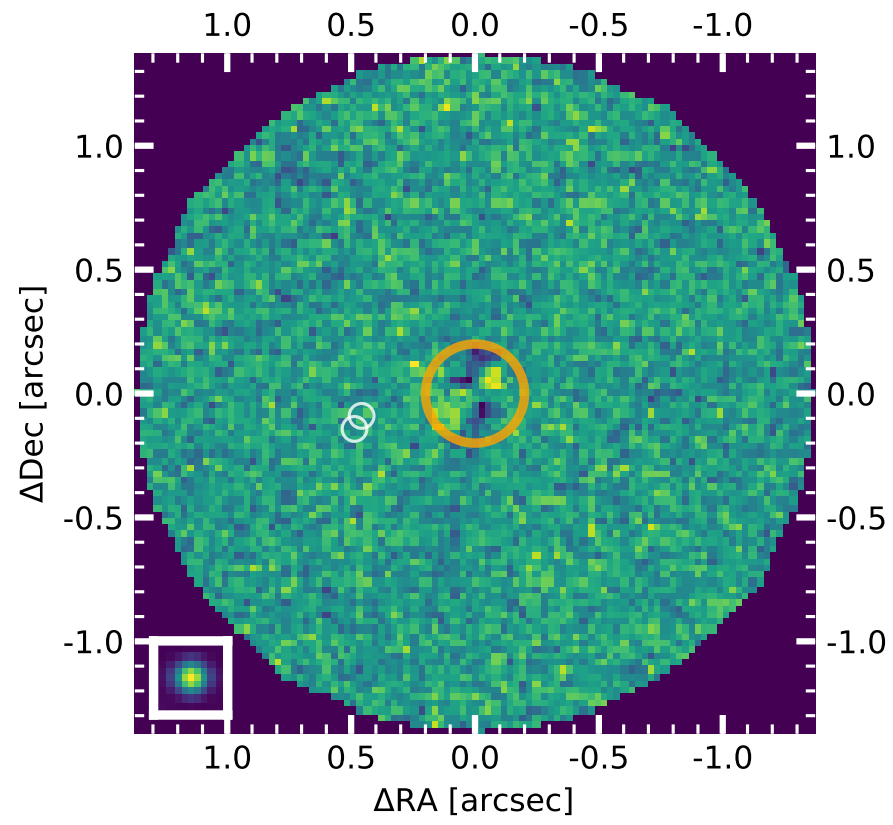

Fig. 2. Image at $L^{\prime}$ band towards J1407. The star is in the middle of the field of view. The two white circles indicate the location of the ALMA source if it is a distant unrelated source and if it has proper motion consistent with an eclipse in 2007. Systematic errors and loss of point source sensitivity occurs within 0.2 arcsec of J1407 (orange circle). No significant point sources are detected around the star.

\section{Astrometric analysis}

There is no source detected with ALMA and NACO within 100 milliarcsec of the star J1407, which would be consistent with a companion in a bound orbit, as determined by the 2007 eclipse and subsequent non-detections of a repeating eclipse event (Mentel et al. 2018). The $3 \sigma$ upper limit on this ALMA non-detection is $57.6 \mu \mathrm{Jy}$. The detected source is too far away from the star to be gravitationally bound to the star (the escape velocity from the star at this separation is a small fraction of the derived transverse velocity), but its position is marginally consistent with a free-floating object that transited J1407 in 2007 and is moving with a transverse velocity of $35 \mathrm{~km} \mathrm{~s}^{-1}$ relative to J1407 (assuming source is co-distant with J1407) derived from the 2007 observations (Kenworthy \& Mamajek 2015), as indicated by the white circle in Fig. 1. Upper and lower uncertainties on the transverse velocity location are shown by the dashed circles. The Sco-Cen association and its subgroups are unbound Wright \& Mamajek (2018) with a 3D velocity dispersion in UCL region of $2.45 \mathrm{~km} \mathrm{~s}^{-1}$, leading to an implied 1D velocity dispersion of $1.4 \mathrm{~km} \mathrm{~s}^{-1}$ at the location of J1407, meaning that this object is unbound from the association and will leave it on a timescale of several million years, presumably having been ejected from one of the stellar systems in the association. 


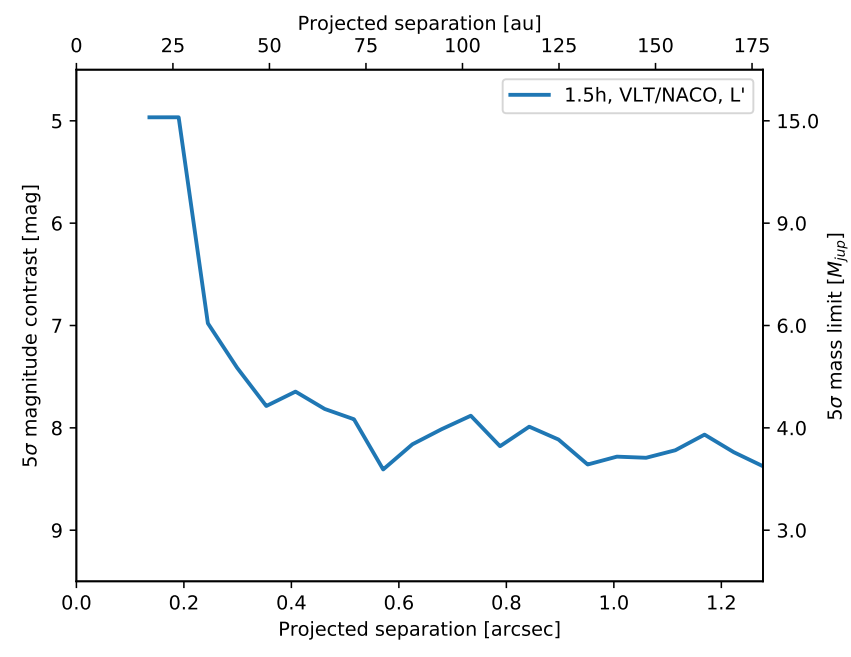

Fig. 3. Contrast curve for NACO $L^{\prime}$ observations towards J1407. The curve shows the $5 \sigma$ point source limit and uses the AMES-Cond model to convert from apparent magnitude to planetary mass.

The measured brightness of the point source is consistent with our models for a ring system with a ring particle size greater than $100 \mu \mathrm{m}$ (see next section), but the distance between the ALMA source and the location of J1407 in 2007 is $438 \pm$ 8 mas, which is equal to a separation of 61 au at the distance of J1407. We have spent the months since the observations confirming with both the ALMA Allegro node in Leiden and independently with our own reduction pipelines that the astrometry is consistent, and so we adopt this astrometric solution for the rest of the paper.

\section{Modelling of a ring system at millimetre wavelengths}

The optical eclipse of 2007 was seen at broad-band optical wavelengths, with no colour information obtained during the eclipse. Analysis of the optical light curve show sharp transitions in optical depth, implying the presence of discontinuities in particle density with ring radius, very similar to the rings of Saturn. This indicates that the rings are geometrically thin (with a height-todiameter ratio $h_{\text {ring }} / d_{\text {ring }} \approx 0.001$; Mamajek et al. 2012) We note that this estimate is only valid where large gradients in the light curve are seen; during the time of smallest projected separation of J1407 behind the disk system, the derived height-to-diameter ratio can be much higher as the path of the star is then tangential to any azimuthally symmetrical structure in the disk (see Fig. 3 in Kenworthy \& Mamajek 2015). Both of these findings point to a system in which the relative velocities between the particles may be small, leading to dust collisions that are not destructive but build up to larger sizes. This is also seen in Saturn's rings where the dust particles are millimetre to centimetre sized (e.g. Tiscareno 2013). To model the continuum emission from the ring system, we adopt the model from Baraffe et al. (2008), which is closest to the mass and age constraints and where the ring-hosting object has a mass $5 M_{\text {Jup }}$, age $16.67 \mathrm{Myr}$, radius $1.337 R_{\text {Jup }}$, and luminosity $\log _{10}\left(L_{\text {planet }} / L_{\odot}\right)=-4.69$.

The flux from the ring system is dependent on the size distribution of the dust particles. We model the dust in the ring system using a 3D version of the radiative transfer code MCMax (Min et al. 2009) for several different input grain size distributions. Models for the luminosity of the central substellar object are taken from Baraffe et al. (2008) for an age of $16 \mathrm{Myr}$.
Thick

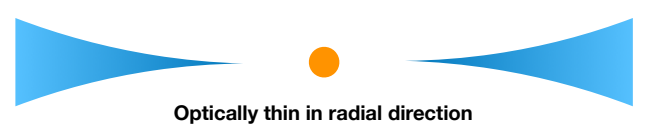

Thin

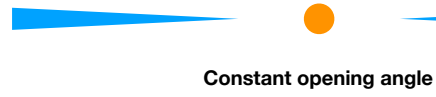

Flared

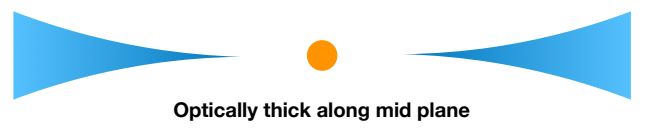

Fig. 4. Diagrams of the three models for the dust (blue) around a central low mass substellar companion (orange). See text for detailed descriptions of the models.

Surface density distribution. We assume an outer radius of 0.6 au for the ring system (equivalent to the Hill radius derived above) and the number density of the dust particles is adjusted to match the optical depth measured at optical wavelengths for each of the rings from the model in Kenworthy \& Mamajek (2015). The surface density is calculated to match the optical depth in the rings as measured: $\Sigma=\tau_{V} / \kappa_{V}$ with $\sigma$ the surface density, $\tau_{V}$ the optical depth in the visible, and $\kappa_{V}$ the mass absorption cross section in the visible. The opacities are computed using irregularly shaped grains (applying the method by Min et al. $2005)$ composed of a mixture of silicate and ice $(40 \% \mathrm{Mg}$ rich pyroxene, $60 \%$ water ice). This is consistent with the amount of water and silicate that would form from a mixture of elements with solar composition, using up all the $\mathrm{Si}, \mathrm{Mg}$, and $\mathrm{O}$. We note that we do not model any dust at radii smaller than $0.19 \mathrm{au}$, which was the smallest projected separation derived in Kenworthy \& Mamajek (2015). Our model represents a lower bound on the amount of dust available for heating in this system. The parameter space for ring models is explored by varying the separation of the ring system from the star (from 5 to $50 \mathrm{au}$ ), varying the luminosity of the substellar object at the centre of the rings, and using several different configurations for the dust in the rings.

Vertical distributions. We chose three configurations of the distribution of dust around the central object and these are shown in Fig. 4. The "Thin" model has a disk whose scale height scales linearly with $R$ as $H=0.005 R$ au, so that along the midplane of the disk the dust is optically thick and self-shields flux from the central object. This is to test the hypothesis that the disk is dynamically cold and highly coplanar, consistent with the assumption that $h_{\text {ring }} / d_{\text {ring }} \approx 0.001$ is valid for all of the disk and not just along the chord sampled by the 2007 transit. The "Thick" model distributes the dust in a flared disk around the substellar object, with a scale height $H$ that increases with distance from the central source $R$ as $H=0.02 R^{1.1}$ au. The temperature structure is computed assuming the disk is optically thin, which provides the brightest possible disk at millimetre wavelengths. The dust distribution in the "Flared" model is identical to that in the Thick model, but the dust is optically thick along the midplane of the disk. Heating from the primary star J1407 and from the companion $\mathrm{J} 1407 \mathrm{~b}$ are taken into account using the stellar parameters listed in van Werkhoven et al. (2014) and 
Kenworthy et al. (2015), and for a range of semi-major separations of the ring system from the central star.

\section{Hypotheses}

We discuss four hypotheses to explain our results:

- the ring system is in a bound orbit around the star J1407, and we have an upper limit from the ALMA photometry;

- the detected ALMA source is a ring system that is gravitationally unbound from (and possibly completely unrelated to) the star J1407;

- the ALMA source is a background galaxy unrelated to the star or 2007 occulting object;

- the source is a noise peak in the synthesised image.

\subsection{An upper limit on a ring system around J1407}

If we assume that the ALMA source is not the ring system detected in 2007, then the ring system is in orbit around J1407 and we do not detect it with the ALMA observations. The models shown in Fig. 5 are generated for different dust geometries and for different separations from the parent star J1407, along with the upper limit at the location of J1407. The three different colours represent different distances of the ring system from the star: 5, 10, and 15 au for yellow, orange, and blue, respectively. Neither the Thin nor the Flaring model is constrained by the observations. The emitted flux increases for larger grain sizes, and we could detect a Thick model disk for all masses above 20 Jupiter masses and particle sizes greater than $1 \mathrm{~mm}$.

\subsection{A free-floating ring system}

We are looking towards a young stellar association and so there is the possibility that we are seeing a young planet ejected from elsewhere in the association. The angular separation of the ALMA source from the location of the star $(438 \pm 8$ mas) is consistent with the expected separation for an object that has travelled at $35 \mathrm{~km} \mathrm{~s}^{-1}$ since the occultation in 2007 $(543 \pm 82$ mas), which we indicate with the solid white circle in Fig. 1 and dotted white circles marking the associated uncertainty. This transverse velocity was derived in Kenworthy et al. (2015) by analysis of the steepest gradients in the light curve of the 2007 eclipse and considering a straight-edged opaque occulter moving across the central disk of the star with a velocity perpendicular to the edge of the occulter. The transverse velocity is then proportional to the rate of change of stellar flux and the diameter of the star; knowing the light curve gradient and diameter of the star gives the velocity.

The discussion in Kenworthy et al. (2015) showed that several gradients would have to be discarded to make a closed circular orbit work for the orbit of the ring system, and instead an eccentric orbit was strongly preferred, or that the system could be on an unbound hyperbolic orbit with coincidental alignment in 2007. Looking at the NACO data, any ring system would have a central object with a mass less than $6 M_{\text {Jup }}$, which would be entirely possible for an unbound ring system since an unbound object does not have an associated Hill sphere.

This could be the first direct detection of the ring system that eclipsed the star J1407 in 2007, and from the models in Fig. 5 we can determine that the substellar object has to have a mass $>6 M_{\text {Jup }}$ for a fixed particle size of $1 \mathrm{~mm}$ in the Thick model case, or that the particle size is larger than $1 \mathrm{~mm}$ and the substellar object can be considerably lower in mass. If this is also the object that occulted J1407 in May 2007, the transverse velocity is
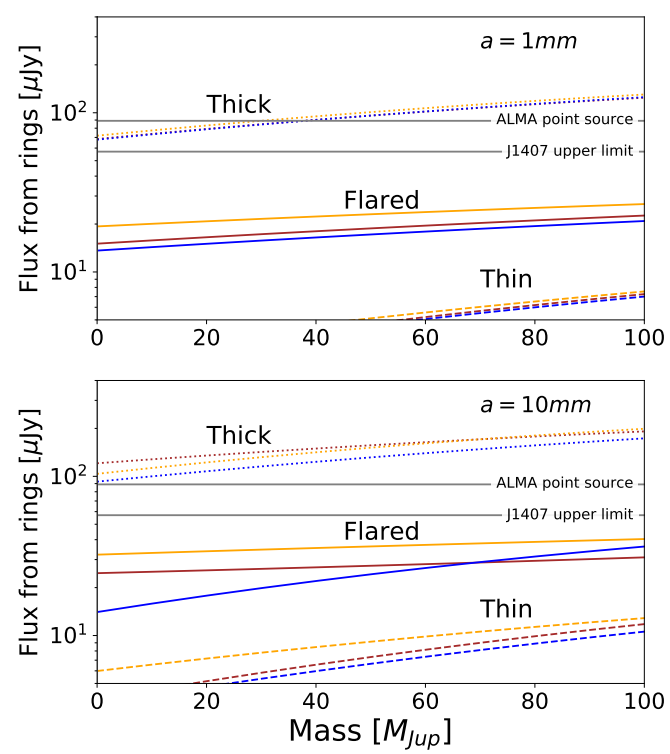

Fig. 5. Models for the sub-mm dust emission from the ring system at ALMA Band 7 as a function of mass of the central substellar object using models from Baraffe et al. (2008), together with the ALMA photometry of the point source and the upper limit at the location of the star J1407. Three different disk configurations are presented. Colours correspond to separations from the star J1407: yellow $5 \mathrm{au}$, orange $10 \mathrm{au}$, blue 16 au.

$35 \mathrm{~km} \mathrm{~s}^{-1}$, which is considerably higher than the escape velocity of the Sco-Cen cluster. It therefore seems highly unlikely that we are seeing a young planet in the process of being ejected from the cluster.

With this hypothesis, we predict that the ALMA source has a

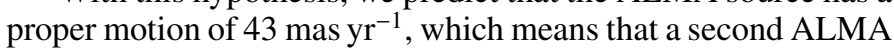
epoch measurement in 2020 will show approximately 130 mas of total proper motion along the line passing through J1407 and the 2017 ALMA source.

\subsection{An unrelated background source}

Using the galaxy counts measured at $1.3 \mathrm{~mm}$ with ALMA from Aravena et al. (2016) we estimate the source background count at $850 \mu \mathrm{m}$ (Band 7) by estimating the number counts at $1.2 \mathrm{~mm}$ and then converting to $850 \mu \mathrm{m}$ number counts. In Aravena et al. (2016), the flux $S$ in different bands is given as $S_{1.2 \mathrm{~mm}}=$ $0.4 S_{870 \mu \mathrm{m}}=0.8 S_{1.1 \mathrm{~mm}}$. Taking the mean of the two shorter wavelengths gives $S_{1.2 \mathrm{~mm}}=0.6 S_{850 \mu \mathrm{m}}$. Our source has $S_{850 \mu \mathrm{m}}=$ $0.1 \mathrm{mJy}$ so $S_{1.2 \mathrm{~mm}}=0.06 \mathrm{mJy}$ for our ALMA detection.

Using Table 2 from Aravena et al. (2016) we estimate 71500 sources with a flux of $89 \mu \mathrm{Jy}$ or greater per square degree. The probability of finding more than zero galaxies within a radius of 0.5 arcsec is around 0.0047 , or 1 in 212 , and the probability of finding a galaxy within the annulus representing a $35 \mathrm{~km} \mathrm{~s}^{-1}$ free-floating object is 0.0029 , or 1 in 341 . It seems unlikely that the source is a background galaxy, but we cannot rule this hypothesis out without a second epoch observation with ALMA.

\subsection{A noise fluctuation}

The last hypothesis we consider is that the ALMA source is a noise fluctuation. This is the brightest peak within the $50 \%$ power point of the synthesised ALMA field of view (and certainly within the proper motion circle). Our analysis uses the primary beam corrected images, which means the noise increases 
away from the phase centre, but the detection is close enough to the phase centre that this should not impact the derived statistical significance. A detection at a second epoch would increase confidence in our interpretation.

\section{Conclusions and future work}

In this paper we present observations using ALMA and NACO towards the young star J1407 in order to determine the nature of the object that caused the light curve seen in May 2007, and we report the detection of an unresolved source consistent with dust heated by a central substellar object. We present several hypotheses for the detected ALMA source and we note that it is at an angular separation consistent with a free-floating ring system that happened to move through the line of sight between us and J1407 in May 2007. The upper limit of $6 M_{\text {Jup }}$ at the location of the ALMA source implies that we are seeing a free-floating ring system around an exoplanet, assuming the ring system formed at the same time as Sco-Cen. If the ALMA source is the object which occulted J1407 in 2007, then the proper motion of the ALMA source is 43 mas $\mathrm{yr}^{-1}$. An observation with ALMA three or more years after the 2017 detection will show this proper motion with enough significance to confirm or rule out this hypothesis, or it will confirm that the source is a distant background object. Further searches are being carried out for other large ring systems both in archival data and in current wide field surveys.

Acknowledgements. We thank the referee for comments and suggestions which improved this paper, and several discussions with the ALMA Allegro node in Leiden for understanding the astrometric calibration of ALMA. This pape makes use of the following ALMA data: ADS/JAO.ALMA\#016.1.00470.S ALMA is a partnership of ESO (representing its member states), NSF (USA) and NINS (Japan), together with NRC (Canada), MOST and ASIAA (Taiwan), and KASI (Republic of Korea), in cooperation with the Republic of Chile. The Joint ALMA Observatory is operated by ESO, AUI/NRAO and NAOJ. This research made use of Astropy (http://www.astropy.org), a community-developed core Python package for Astronomy (Astropy Collaboration 2013; Price-Whelan et al. 2018) and of Matplotlib (Hunter 2007). This research made use of APLpy, an open-source plotting package for Python (Robitaille \& Bressert 2012; Robitaille 2019). This work has made use of data from the European Space Agency (ESA) mission Gaia (https://www . cosmos.esa.int/gaia), processed by the Gaia Data Processing and Analysis Consortium (DPAC, https://www.cosmos.esa.int/web/gaia/dpac/ consortium). Funding for the DPAC has been provided by national institutions, in particular the institutions participating in the Gaia Multilateral Agreement Part of this research was carried out at the Jet Propulsion Laboratory, California Institute of Technology, under a contract with NASA.

\section{References}

Allard, F., Hauschildt, P. H., Alexander, D. R., Tamanai, A., \& Schweitzer, A. 2001, ApJ, 556, 357

Amara, A., \& Quanz, S. P. 2012, MNRAS, 427, 948

Aravena, M., Decarli, R., Walter, F., et al. 2016, ApJ, 833, 68

Armitage, P. J. 2011, ARA\&A, 49, 195

Astropy Collaboration (Robitaille, T. P., et al.) 2013, A\&A, 558, A33

Bailer-Jones, C. A. L., Rybizki, J., Fouesneau, M., Mantelet, G., \& Andrae, R. 2018, AJ, 156, 58

Baraffe, I., Chabrier, G., Barman, T. S., Allard, F., \& Hauschildt, P. H. 2003, A\&A, 402, 701

Baraffe, I., Chabrier, G., \& Barman, T. 2008, A\&A, 482, 315

Butters, O. W., West, R. G., Anderson, D. R., et al. 2010, A\&A, 520, L10

Canup, R. M., \& Ward, W. R. 2002, AJ, 124, 3404

Gaia Collaboration (Brown, A. G. A., et al.) 2018, A\&A, 616, A1

Hunter, J. D. 2007, Comput. Sci. Eng., 9, 90

Kasper, M., Amico, P., Pompei, E., et al. 2009, The Messenger, 137, 8

Kenworthy, M. A., \& Mamajek, E. E. 2015, ApJ, 800, 126

Kenworthy, M. A., Lacour, S., Kraus, A., et al. 2015, MNRAS, 446, 411

Kley, W., \& Nelson, R. P. 2012, ARA\&A, 50, 211

Lenzen, R., Hartung, M., Brandner, W., et al. 2003, SPIE Conf. Ser., 4841, 944

Mamajek, E. E., Quillen, A. C., Pecaut, M. J., et al. 2012, AJ, 143, 72

Marois, C., Lafrenière, D., Doyon, R., Macintosh, B., \& Nadeau, D. 2006, ApJ, 641, 556

Mawet, D., Milli, J., Wahhaj, Z., et al. 2014, ApJ, 792, 97

McMullin, J. P., Waters, B., Schiebel, D., Young, W., \& Golap, K. 2007, ASP Conf. Ser., 376, 127

Mentel, R. T., Kenworthy, M. A., Cameron, D. A., et al. 2018, A\&A, 619, A157

Min, M., Hovenier, J. W., \& de Koter A. 2005, A\&A, 432, 909

Min, M., Dullemond, C. P., Dominik, C., de Koter, A., \& Hovenier, J. W. 2009, A\&A, 497, 155

Perez, S., Dunhill, A., Casassus, S., et al. 2015, ApJ, 811, L5

Pérez, S., Marino, S., Casassus, S., et al. 2019, MNRAS, 488, 1005

Pojmanski, G. 2002, Acta Astron., 52, 397

Price-Whelan, A. M., Sipőcz, B. M., Günther, H. M., et al. 2018, AJ, 156, 123

Rieder, S., \& Kenworthy, M. A. 2016, A\&A, 596, A9

Robitaille, T. 2019, https: //doi .org/10.5281/zenodo. 2567476

Robitaille, T., \& Bressert, E. 2012, Astrophysics Source Code Library [record ascl: 1208.017]

Rousset, G., Lacombe, F., Puget, P., et al. 2003, SPIE Conf. Ser., 4839, 140

Stolker, T., Bonse, M. J., Quanz, S. P., et al. 2019, A\&A, 621, A59

Sutton, P. J. 2019, MNRAS, 486, 1681

Szulágyi, J., Morbidelli, A., Crida, A., \& Masset, F. 2014, ApJ, 782, 65

Szulágyi, J., Mayer, L., \& Quinn, T. 2017, MNRAS, 464, 3158

Tiscareno, M. S. 2013, in Planets, Stars and Stellar Systems: Solar and Stellar Planetary Systems, eds. T. D. Oswalt, L. M. French, \& P. Kalas (Dordrecht: Springer Science and Business Media), 3, 309

van Werkhoven, T. I. M., Kenworthy, M. A., \& Mamajek, E. E. 2014, MNRAS, 441,2845

Williams, J. P., \& Cieza, L. A. 2011, ARA\&A, 49, 67

Wright, N. J., \& Mamajek, E. E. 2018, MNRAS, 476, 381

Zanazzi, J. J., \& Lai, D. 2017, MNRAS, 464, 3945 